\title{
A smaller heart-aorta-angle associates with ascending aortic dilatation and increases wall shear stress
}

\author{
S. Petteri Kauhanen ${ }^{1,2} \cdot$ Timo Liimatainen $^{3,4} \cdot$ Elina Kariniemi $^{2,5} \cdot$ Miika Korhonen $^{2} \cdot$ Johannes Parkkonen $^{6}$. \\ Juska Vienonen $^{6} \cdot$ Ritva Vanninen ${ }^{2,6} \cdot$ Marja Hedman $^{2,7}$
}

Received: 9 February 2020 / Revised: 10 March 2020 / Accepted: 31 March 2020 / Published online: 22 April 2020

(C) The Author(s) 2020

\begin{abstract}
Objectives The aim of this study was to evaluate whether the orientation of the heart, measured as an angle between the long axis of the heart and ascending aorta midline (heart-aorta-angle, HAA), associates with ascending aortic (AA) dilatation. Furthermore, the association between HAA and wall shear stress (WSS) was studied.

Methods HAA was retrospectively measured in 1000 consecutive coronary artery computed tomographic angiography (CCTA) images in patients with low-to-moderate pretest probability for coronary artery disease (CAD). To evaluate the effects of HAA on AA flow, 4D flow MRI was performed for 28 patients with AA dilatation $(>40 \mathrm{~mm})$ and WSS was analyzed.

Results The mean age of patients undergoing CCTA was $52.9 \pm 9.8$ years; $66.5 \%$ were women. Their median HAA was $128.7^{\circ}$ and interquartile range $123.3-134.1^{\circ}$. HAA was significantly smaller in patients with dilated AA (median $126.7^{\circ}\left[121.3-130.8^{\circ}\right]$ ) compared with the patients with normal AA (median $\left.129.5^{\circ}\left[124.3-135.3^{\circ}\right], p<0.001\right)$. HAA was smaller in males $(p<0.001)$ and in patients with diabetes $(p=0.016)$, hypertension $(p=0.001)$, CAD $(p=0.003)$, hypercholesterolemia $(p<0.001)$, and bicuspid aortic valve $(p=0.025)$ than without these factors. In a subpopulation without any of these underlying diseases $(n=$ 233), HAA was still significantly smaller in the patients with dilated AA (median $127.9^{\circ}\left[124.3-134.3^{\circ}\right]$ ) compared with patients with normal AA (median $131.9^{\circ}\left[127.6-136.9^{\circ}\right], p=0.013$ ). In 4D flow MRI, a smaller HAA correlated with increased total WSS in the outer curvature of the proximal AA $(r=-0.510, p=0.006)$.

Conclusion A smaller HAA associates with AA dilatation and affects the blood flow in the proximal AA.

Key Points

- A smaller angle between the long axis of the heart and ascending aorta midline associated with ascending aortic dilatation.

- A smaller heart-aorta-angle correlated with increased total wall shear stress in the outer curvature of the proximal ascending aorta.
\end{abstract}

Keywords Aorta thoracic $\cdot$ Aortic aneurysm $\cdot$ Heart ventricles $\cdot$ Tomography $\mathrm{x}$-ray computed $\cdot$ Magnetic resonance imaging

\begin{tabular}{|c|c|c|c|}
\hline \multicolumn{2}{|c|}{ Abbreviations } & BAV & Bicuspid aortic valve \\
\hline AA & Ascending aorta & BSA & Body surface area \\
\hline AHI & Aortic height index & CAD & Coronary artery disease \\
\hline
\end{tabular}

S. Petteri Kauhanen petkau@student.uef.fi

1 Doctoral Programme of Clinical Research, University of Eastern Finland, Kuopio, Finland

2 Department of Clinical Radiology, Clinical Imaging Center, Kuopio University Hospital, Kuopio, Finland

3 Research Unit of Medical Imaging, Physics and Technology, University of Oulu, Oulu, Finland
4 Department of Diagnostic Radiology, Oulu University Hospital, Oulu, Finland

5 Department of Clinical Physiology and Nuclear Medicine, Clinical Imaging Center, Kuopio University Hospital, Kuopio, Finland

6 School of Medicine, Clinical Radiology, University of Eastern Finland, Kuopio, Finland

7 Department of Cardiothoracic Surgery, Heart Center, Kuopio University Hospital, Kuopio, Finland 


\section{CCTA Coronary artery computed tomography angiography \\ CT Computed tomography \\ ESC European Society of Cardiology \\ HAA Heart-aorta-angle \\ ICC Intraclass correlation coefficients \\ IQR Interquartile range \\ LV Left ventricle \\ MRI Magnetic resonance imaging \\ TRUFI True fast imaging with steady-state precession \\ WSS Wall shear stress}

\section{Introduction}

The diameter of thoracic aorta has been shown to increase with aging, male gender, and increased body surface area (BSA) [1]. Furthermore, ascending aortic (AA) dilatation is associated with conventional cardiovascular risk factors, such as hypertension and smoking [2-4]. Also, the length of the thoracic aorta has been shown to be related to age; i.e., aortic elongation may be a part of the normal aging process. Adriaans et al have demonstrated that the thoracic aortic length increases by $59-66 \mathrm{~mm}$ between the ages of 20 and 80 years [5].

However, the associations between AA dilatation and the angle between the heart and AA (the heart-aortaangle, HAA) have not been studied previously. Under normal conditions, the heart is oriented with the right ventricle on the anterior side and the left atrium on the posterior side [6]. The axis of the heart is orientated at approximately $45^{\circ}$ to the left of an anteroposterior line drawn from the spine to the anterior chest wall [7]. It has been described that the heart is oriented vertically downwards in the "Valentine" position, which means that the heart is a solitary organ and provides no reference point for its location within the chest [8]. In healthy subjects, the HAA has been reported to be approximately $140 \pm 7^{\circ}$ [9].

In patients with AA dilatation, the aortic blood flow has been shown to be displaced even in the case of normal tricuspid aortic valve [10]. Furthermore, the displaced flow may lead to increased wall shear stress (WSS) on the displaced side of the AA [10]. A bicuspid aortic valve (BAV) and aortic stenosis have also been shown to associate with increased WSS values [11].

The purposes of this study were (1) to investigate the association between HAA and AA dilatation and (2) to analyze whether the HAA has an influence on the blood flow and WSS in the AA.

\section{Methods}

The study was approved by the Ethics Committee, Hospital District of Northern Savo. Coronary artery computed tomography angiography (CCTA) imaging was performed on the basis of clinical indications; thus, the patients were not exposed to additional radiation dose. The patients' clinical treatment was unaffected by the study. The populations of the present study have also been analyzed in the prior publications $[10,12,13]$.

\section{Patient population}

\section{CCTA patient population}

This retrospective study examined 1065 consecutive patients with low-to-moderate pretest probability for coronary artery disease (CAD) and without pre-existing aortic disease scheduled for CCTA in Kuopio University Hospital between January 2012 and March 2018. Sixty-four patients were excluded due to motion artifacts or inadequate visibility of AA in CCTA images and one patient who was aged under 16 years. The mean age of the CCTA study population $(n=1000)$ was $52.9 \pm 9.8$ years and the majority of the patients were women $(n=665,66.5 \%)$. Patients' baseline characteristics are presented in Table 1.

\section{D flow MRI patient population}

The power calculation was performed for the Spearman test with power 0.8 and obtained $p$ value and significance level 0.05 . Based on the results of power calculation, this prospective study included 28 patients with AA dilatation who were imaged with aortic magnetic resonance imaging (MRI) combined with 4D flow analysis between August 2017 and December 2019 in Kuopio University Hospital and who had prior thoracic CT scans. All patients had normal tricuspid anatomy of the aortic valve without aortic stenosis. Aortic dimensions were measured from the MRI images and the previously performed thoracic CT scans were used for the measurement of HAA. The mean age of 4D flow MRI population $(n=28)$ was $65.6 \pm 6.3$ years and $25(89.3 \%)$ of patients were male. Patients' baseline characteristics are presented in Table 1.

\section{CCTA imaging}

CCTA imaging was performed during mid-diastole according to the routine clinical practice using four different $\mathrm{CT}$ scanners capable of ECG-gated fast coronary CT imaging (Somatom Definition AS 64; Somatom Definition AS+ 128; Definition Edge; and Definition Flash, Siemens Medical Solutions). Collimation was $64 \times 0.6 \mathrm{~mm}$ with the Somatom Definition 
Table 1 Baseline characteristics of the CCTA and 4D flow MRI population. BSA, body surface area; $C A D$, coronary artery disease; CCTA, coronary computed tomography angiography

\begin{tabular}{|c|c|c|c|c|c|c|}
\hline & \multicolumn{3}{|l|}{ CCTA population } & \multicolumn{3}{|c|}{ 4D flow MRI population } \\
\hline & All patients, $n=1000$ & Males, $n=335$ & Females, $n=665$ & All patients, $n=28$ & Males, $n=25$ & Females, $n=3$ \\
\hline Age (years) & $52.9 \pm 9.8$ & $48.5 \pm 10.8$ & $55.1 \pm 8.5$ & $65.6 \pm 6.3$ & $65.3 \pm 6.5$ & $68.3 \pm 3.8$ \\
\hline Height $(\mathrm{cm})$ & $168.7 \pm 9.6$ & $178.3 \pm 6.3$ & $163.6 \pm 6.6$ & $177.6 \pm 7.1$ & $179.2 \pm 5.4$ & $164.3 \pm 6.0$ \\
\hline Weight (kg) & $80.1 \pm 17.7$ & $90.7 \pm 16.1$ & $74.3 \pm 15.8$ & $91.7 \pm 16.6$ & $94.6 \pm 14.9$ & $69.0 \pm 12.1$ \\
\hline $\operatorname{BSA}\left(\mathrm{m}^{2}\right)$ & $1.9 \pm 0.2$ & $2.1 \pm 0.2$ & $1.8 \pm 0.2$ & $2.1 \pm 0.2$ & $2.2 \pm 0.2$ & $1.8 \pm 0.2$ \\
\hline Diabetes & $80(8.0)$ & $30(9.0)$ & $50(7.5)$ & $5(17.9)$ & $4(16.0)$ & $1(33.3)$ \\
\hline Hypertension & $445(45.5)$ & $143(42.7)$ & $312(46.9)$ & $23(82.1)$ & $20(80.0)$ & $3(100.0)$ \\
\hline Hypercholesterolemia & $500(50.0)$ & $160(47.8)$ & $340(51.1)$ & $12(42.9)$ & $10(40.0)$ & $2(66.7)$ \\
\hline Positive family history for CAD & $572(57.2)$ & $168(50.1)$ & $404(60.8)$ & $3(10.7)$ & $2(8.0)$ & $1(33.3)$ \\
\hline Smoking & $254(25.4)$ & $123(36.7)$ & $131(19.7)$ & $3(10.7)$ & $3(12.0)$ & 0 \\
\hline Normal CCTA & $625(62.5)$ & $180(53.7)$ & $445(66.9)$ & - & - & - \\
\hline Over $50 \%$ stenosis in CCTA & 149 (14.9) & $55(16.4)$ & $94(14.1)$ & - & - & - \\
\hline Coronary calcification in CCTA & $226(22.6)$ & $100(29.9)$ & $126(18.9)$ & - & - & - \\
\hline Bicuspid aortic valve & $31(3.1)$ & $22(6.6)$ & $9(1.4)$ & 0 & 0 & 0 \\
\hline Mechanical aortic valve & $1(0.1)$ & 0 & $1(0.2)$ & 0 & 0 & 0 \\
\hline
\end{tabular}

AS 64 and $128 \times 0.6 \mathrm{~mm}$ for the other scanners. The specific imaging procedure has been presented in a previous study [12].

\section{Magnetic resonance imaging}

MRI was performed with a Siemens Magnetom Aera 1.5-T scanner. MRI angiography was performed without contrast media with true fast imaging with steady-state precession (TRUFI) with a respiratory navigator. 4D flow MRI was performed with ECG-gating and free-breathing without contrast media. The imaging parameters were selected in line with the 4D flow consensus statement [14]. The detailed imaging procedure has been presented in a previous study [10].

\section{Data assessment}

One experienced observer (S.P.K.) retrospectively analyzed the CCTA images on an IDS7 diagnostic workstation (version 17.3.6; Sectra Imtec). The AA was divided into 3 planes: sinus valsalva, sinotubular junction, and mid-AA. According to the current international recommendations, aortic diameters were measured from the outer-to-outer vascular wall perpendicular to the centerline of the vessel [1]. Aortic valve anatomy (tricuspid, bicuspid, or mechanical aortic valve prosthesis), middle diastolic diameter of cardiac left ventricle (LV), area of left atrium, thickness of left ventricular posterior wall, and interventricular septum were registered. The thickness of intraventricular septum was further dichotomized as under the mean value and over the mean value.

The HAA was measured in multiplanar reformatted images as described earlier in the literature [9]. The 3-chamber projection was used to draw the midline through the LV from the apex through the center of the mitral valve and the midline of the left outflow tract and the AA. The HAA measurement method is presented in Fig. 1. To assess interobserver reproducibility, two observers (S.P.K. and E.K.) independently measured the HAA from 100 CCTA images. To assess the intraobserver reproducibility, one observer (E.K.) repeated the 100 HAA measurements.

\section{AA dilatation classification methods}

Main classification method for aortic dilatation was based on the current (2014) European Society of Cardiology (ESC) guidelines. According to these guidelines, the AA is considered dilated regardless of the gender when its greatest diameter exceeds $40 \mathrm{~mm}$ in any of the three measurement planes [1].

Hannuksela et al proposed age-related formula (upper limit for normal mid-AA $=30 \mathrm{~mm}+0.20 \times$ age) and body sizeadjusted formula, which were selected as additional classification methods $[13,15]$. To assess the body size adjusted formula, aortic height index $(\mathrm{AHI})$ was calculated $\left(\mathrm{AHI}=\frac{\text { Aortic diameter }(\mathrm{mm})}{\text { patient height }(\mathrm{m})}\right)$. The upper limit for normal mid-AA was set as $23.3 \mathrm{~mm} / \mathrm{m}$ according to our prior publication [13]. 


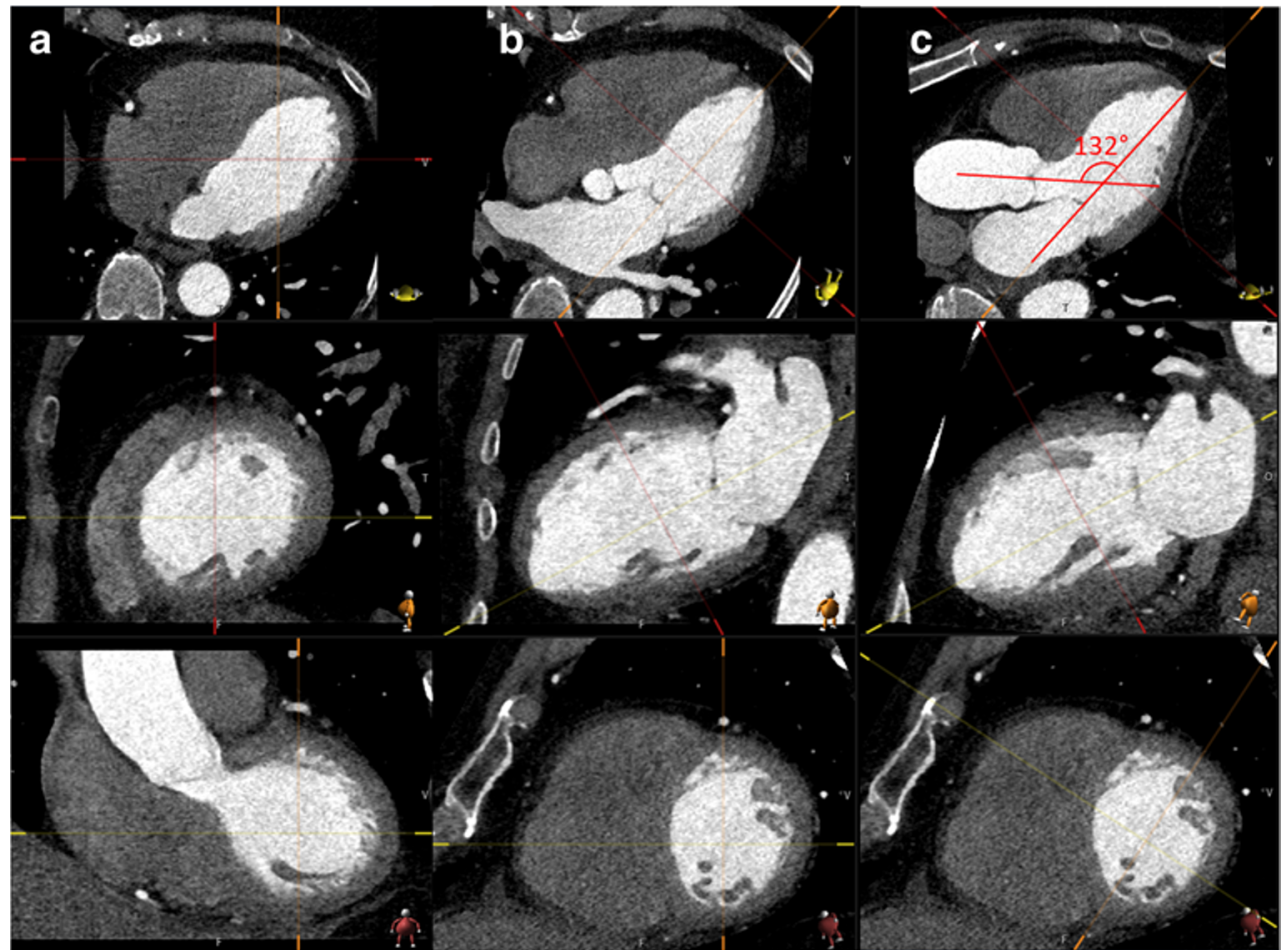

Fig. 1 Multiplanar reformatted images of the heart-aorta-angle (HAA) measurement. An axial direction on the top, a sagittal direction on the middle, and coronal direction on the bottom lines. a The first step was to set the middle of the left ventricle in coronal and sagittal images. b The second step was to set the longitudinal line through the center of the mitral valve annulus and left ventricular apex in axial and sagittal images. $\mathbf{c}$ The

\section{MRI flow parameters}

Circumferential wall shear stress $\left(\mathrm{WSS}_{\mathrm{C}}\right.$, parallel to the emitter plane), axial ( $\mathrm{WSS}_{\mathrm{A}}$, perpendicular to emitter plane), and total WSS $\left(\mathrm{WSS}_{\mathrm{T}}\right.$, geometric sum of $\mathrm{WSS}_{\mathrm{C}}$ and $\mathrm{WSS}_{\mathrm{A}}$ ) were measured at 5 different levels of AA (Fig. 2): (1) sinus valsalva, (2) sinotubular junction, (3) proximal tubular part, (4) mid-AA, and (5) proximal part of the aortic arch. The aortic ring was divided into six $60^{\circ}$ segments. The starting point $\left(0^{\circ}\right)$ was defined to be in the inner curve of AA and segment 1 covering from $0^{\circ}$ to $60^{\circ}$ in a counter-clockwise direction [10]. Peak systolic WSS was obtained for the analysis. Intra- and interobserver reproducibility analyses for the flow parameters of the 4D flow have been previously published and proved to be moderate [10].

\section{Risk factors}

Risk factors for cardiovascular diseases as well as other baseline characteristics were collected from the medical records. The patient was defined as hypertensive if he/she was receiving medication for hypertension and as diabetic and if thepatient had two separate fasting plasma glucose levels $\geq 7.0 \mathrm{mmol} / 1$ or $\geq 11 \mathrm{mmol} / \mathrm{l}$ in a glucose tolerance test or last step was to take the 3-chamber projection by turning the short axis line anticlockwise in coronal images so that AA become present and measured the angle between the left ventricle long axis line and middle AA line. The HAA was $132^{\circ}$ in this case. The HAA was measured according to the previous report [9]

$\mathrm{HbA}_{1 \mathrm{C}} \geq 48 \mathrm{mmol} / \mathrm{l}$. Current smokers and those who had stopped continuous smoking less than 30 years previously were considered smokers. Based on the coronary artery findings in the CCTA, the patients were dichotomized as positive or negative in terms of CAD. Hypercholesterolemia was determined according to the Finnish national recommendations as high LDL ( $>3 \mathrm{mmol} / \mathrm{l}$ ) and low HDL (males $<1 \mathrm{mmol} / \mathrm{l}$, females $<1.2 \mathrm{mmol} / \mathrm{l}$ ) concentrations as described previously [12]. Body mass index was dichotomized as normal weight $\left(<25 \mathrm{~kg} / \mathrm{m}^{2}\right)$ or as overweight or obesity $\left(\geq 25 \mathrm{~kg} / \mathrm{m}^{2}\right)[16]$. Age was dichotomized as under the mean value or over the mean value.

\section{Statistical analysis}

The normality of the HAA data was analyzed using the Kolmogorov-Smirnov test. Skewed distributed parameters were tested with the Mann-Whitney $U$ test, the results being presented as median and interquartile range (IQR). Correlations between the HAA and continuous scaled parameters were tested by using the Spearman correlation test. Multivariate logistic regression was used to test the association between HAA and different classification methods of AA dilatation. 
Fig. 2 a Wall shear stress was analyzed in 5 planes of ascending aorta: (1) sinus valsalva, (2) sinotubular junction, (3) proximal part of tubular aorta, (4) midascending aorta, and (5) proximal part of the aortic arch. b The aortic ring was divided into six segments (each $60^{\circ}$ ). The red arrow points to the inner curvature of AA indicating the zero point of the WSS measurements

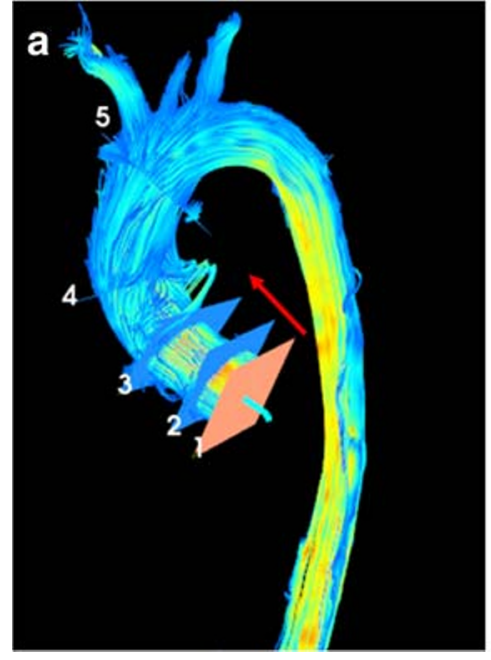

b

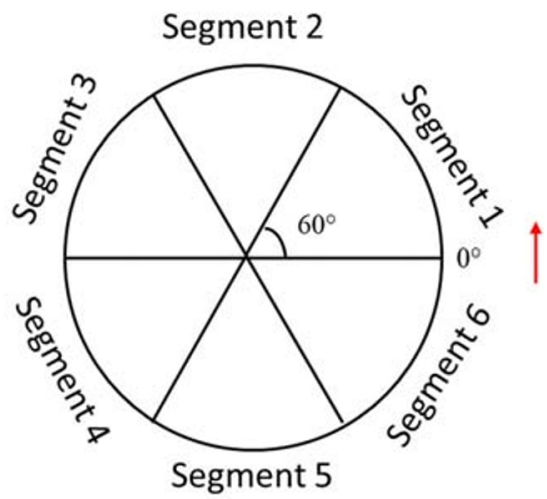

Paired samples $t$ test was used to test the systematic error in intra-and interobserver analyses. Intraclass correlation coefficients (ICCs) using a two-way mixed effects model with absolute agreement were used to calculate intra- and interobserver reproducibility. ICC values from 0.0 to 0.2 were considered negligible, from 0.2 to 0.4 very low, from 0.4 to 0.7 moderate, from 0.7 to 0.9 strong, and from 0.9 to 1.0 very strong.

Statistical significance was set to $p<0.05$ and high statistical significance to $p<0.001$. All statistical analyses were performed using the IBM SPSS Statistics 25. Statistical analysis was performed in collaboration with a biostatistician.

\section{Results}

According to the ESC guidelines, 230 patients (23\%) were stratified as having AA dilatation in the CCTA population when the measurement results from all three levels were combined.

The median HAA was $128.7^{\circ}\left(123.3-134.1^{\circ}\right)$ and the mean HAA was $128.8 \pm 8.1^{\circ}$ in the CCTA patient population. By using the ESC 2014 guidelines, smaller HAA values associated significantly with AA dilatation on all measurement levels: in the sinus valsalva level $(p<0.001)$, in sinotubular junction $(p=0.004)$, and in the mid-AA $(p<0.001)$ in all of the patients analyzed together and in males. In females, smaller HAA values were not significantly associated with AA dilatation $(p=0.097-0.507)$. When all 3 planes were combined, HAA was significantly smaller in the patients with dilated AA (median $126.7^{\circ}$, IQR $121.3-130.8^{\circ}$ ) compared with the patients with normal AA (median $129.5^{\circ}$, IQR $124.3-135.3^{\circ}$, $p<0.001$; Table 2 and Fig. 3).

A smaller HAA correlated very weakly with the body sizeadjusted AA diameters $(r=-0.256, p<0.001)$. A smaller HAA associated with AA dilatation also by using agerelated formula being $125.5^{\circ}\left(118.9-130.9^{\circ}\right)$ in patients with dilated AA and $129.0^{\circ}\left(123.7-134.3^{\circ}\right)$ in patients with normal $\mathrm{AA}, p=0.001$, and by using body size-adjusted classification $\left(125.7^{\circ}\left[120.2-131.2^{\circ}\right]\right.$ vs. $\left.128.8^{\circ}\left[123.6-134.2^{\circ}\right], p=0.002\right)$.

A smaller HAA associated with AA dilatation by using the ESC classification $\left(B=-3.1^{\circ}, p<0.001\right)$, but not by agerelated classification $\left(\mathrm{B}=-1.1^{\circ}, p=0.374\right)$ or body sizeadjusted classification $\left(B=-0.8^{\circ}, p=0.562\right)$ in multivariate regression analysis.

When analyzed with 4D flow MRI, smaller HAA correlated significantly with the increased total WSS in the outer curvature of the proximal tubular part of dilated AA ( $r=-$ $0.510, p=0.006$, Fig. 4 ) in the $120^{\circ}$ segment. WSS was not correlated with the smaller HAA in the other planes ( $p=0.121-$ 0.428). Illustrative $4 \mathrm{D}$ flow MRI images of the increased $\mathrm{WSS}_{\mathrm{T}}$ in the outer curvature of AA in patients with smaller and larger HAA are shown in Fig. 5.

Factors that associated with the smaller HAA are collected in Table 3. Conventional risk factors for cardiovascular diseases such as diabetes $(p=0.016)$, hypertension $(p=0.001)$, hypercholesterolemia $(p<0.001)$, and male gender $(p<0.001)$ and the presence of CAD $(p=0.003)$ significantly associated with smaller HAA. The presence of BAV was also associated with smaller HAA $(p=0.025)$. The smaller HAA correlated weakly with BSA $(r=0.213, p<0.001)$, intraventricular septum thickness $(r=0.227, p<0.001)$, and patient's age $(r=0.191, p<0.001)$.

For further analysis, we selected a subpopulation of 233 patients with no risk factors (diabetes, hypertension, hypercholesterolemia, CAD, BAV, or mechanical aortic valve). The majority i.e. $70.0 \%$ of these patients were female and their mean age was $48.3 \pm 11.4$ years. In this subpopulation, the mean HAA was $131.6 \pm 7.5^{\circ}$. According to the ESC guidelines, 35 patients (15.0\%) were stratified as having AA dilatation. Furthermore, $39 \%$ of males in this subpopulation had AA dilatation. When analyzing the subpopulation with no risk 
factors, the smaller HAA was still significantly associated with AA dilatation (median $127.9^{\circ}$, IQR $124.3-134.3^{\circ}$ in the patients with dilated AA compared with median $131.9^{\circ}$, IQR $127.6-136.9^{\circ}$ in the patients with normal AA, $p=0.013$ ).

No systematic errors in the HAA measurements were detected between the two independent observers. Intraobserver reproducibility was very strong $(\mathrm{ICC}=0.914)$ and interobserver reproducibility was strong $(\mathrm{ICC}=0.870)$.

\section{Discussion}

This study aimed to evaluate whether the HAA would be associated with AA dilatation. As a result, we found in a consecutive population of 1000 patients that smaller HAA strongly associated with dilated AA. Apart from the structural information in CCTA images, we performed 4D MRI flow analysis in 28 patients with AA dilatation to clarify if this association could be explained by altered flow conditions in AA. It has been earlier demonstrated that in patients with AA dilatation, the aortic flow is displaced into the outer curve of AA leading to increased WSS [10]. In our study, the patients with AA dilatation and smaller HAA showed significant increase in WSS especially in the outer curvature of the proximal aorta. This finding suggests that the smaller HAA leads to more intense angle between the long axis of the heart and proximal ascending aorta, which might have potential to alter the blood flow in AA and, consequently, could lead to increased WSS making the vessel more susceptible to dilatation.

The association between HAA and AA dilation has not been previously evaluated. Aortic elongation occurs with increasing age, which might have an effect on the position of the heart [5]. Smaller HAAs have associated also with AA dilatation by using age-related formula postulated by Hannuksela et al [15]. Furthermore, heart diseases are associated with changes in myocardial morphology which can further associate to HAA. For example, hypertrophic obstructive cardiomyopathy has been shown to be associated with smaller HAA [9]. Increased thickness of intraventricular septum associated with the smaller HAA also in the present study.

Kwon et al presented that the mean HAA was $140 \pm 7^{\circ}$ in the healthy controls and $128 \pm 10^{\circ}$ in the hypertensive, elderly patients [9]. In the present study, the mean HAA was 131.6 \pm $7.5^{\circ}$ in the subgroup of patients with no risk factors. Our smaller values of the HAA in the "healthy" subgroup might be explained that patients had still low-to-moderate pretest probability for $\mathrm{CAD}$; thus, this subgroup cannot be considered as completely healthy patients. The mean HAA was $128.8 \pm$ $8.1^{\circ}$ in the overall CCTA patient population, paralleling the results of Kwon et al in hypertensive-elderly patients.

Since it is well known that conventional cardiovascular risk factors, such as hypertension, smoking, diabetes, and 
Fig. 3 Representative images of the heart-aorta-angle. a Dilated ascending aorta $(46.8 \mathrm{~mm})$, HAA $119.3^{\circ}$. b Normal ascending aorta (36.7 mm); HAA $132.2^{\circ}$

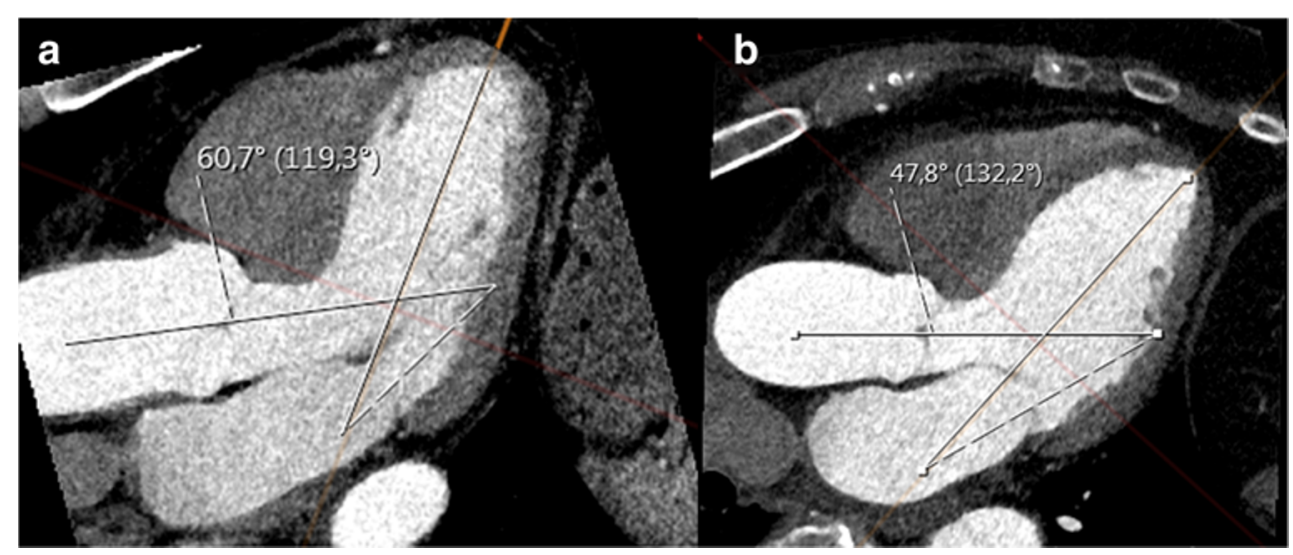

hypercholesterolemia, are also risk factors for AA dilatation [17-19], we examined and excluded these risk factors in a selected subpopulation of 233 patients. Even in this subpopulation without these risk factors, the smaller HAA associated significantly with AA dilatation, suggesting that HAA might constitute an independent risk factor for AA dilatation.

Overweight or obesity was associated with smaller HAA in both genders compared to the patients with normal weight. Typically, upper body obesity is more commonly found in males whereas lower body obesity is more commonly found in females [20]. A smaller HAA was strongly associated with male gender. An increased amount of upper abdominal visceral fat especially in males may push the diaphragm and heart to the altered orientation. Although the correlation between BSA and smaller HAA was relatively weak, obesity remains to be one of the few factors to be interfered by lifestyle and medication. However, smaller HAA correlated with AA diameter when it was adjusted to patient's height and when using the AHI classification method.

The smaller HAA did not reach statistically significant associations with AA dilatation in females. In females, the prevalence of AA dilatation is much lower than that in males when using the ESC dilatation classification [13]. Females have

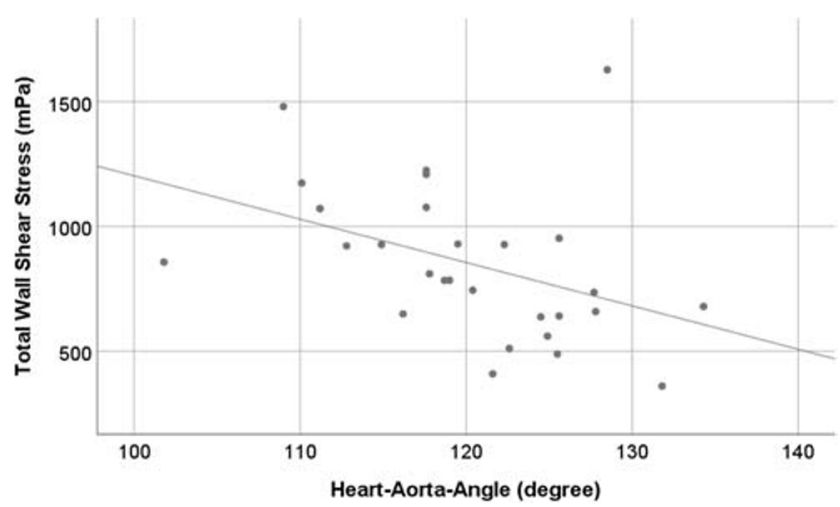

Fig. 4 Correlation between the heart-aorta-angle and the total wall shear stress in the proximal part of tubular aorta $(r=-0.510, p=0.006)$ in the outer curvature of aorta in $120^{\circ}$ segment lesser abdominal obesity than males [20]. Further studies are, however, needed to explain why the associations between smaller HAA and AA dilatation are different in males and females.

The main limitations of this study were the higher fraction of female patients and that only a limited volume of AA was included in the image field-of-view. This was a result from the

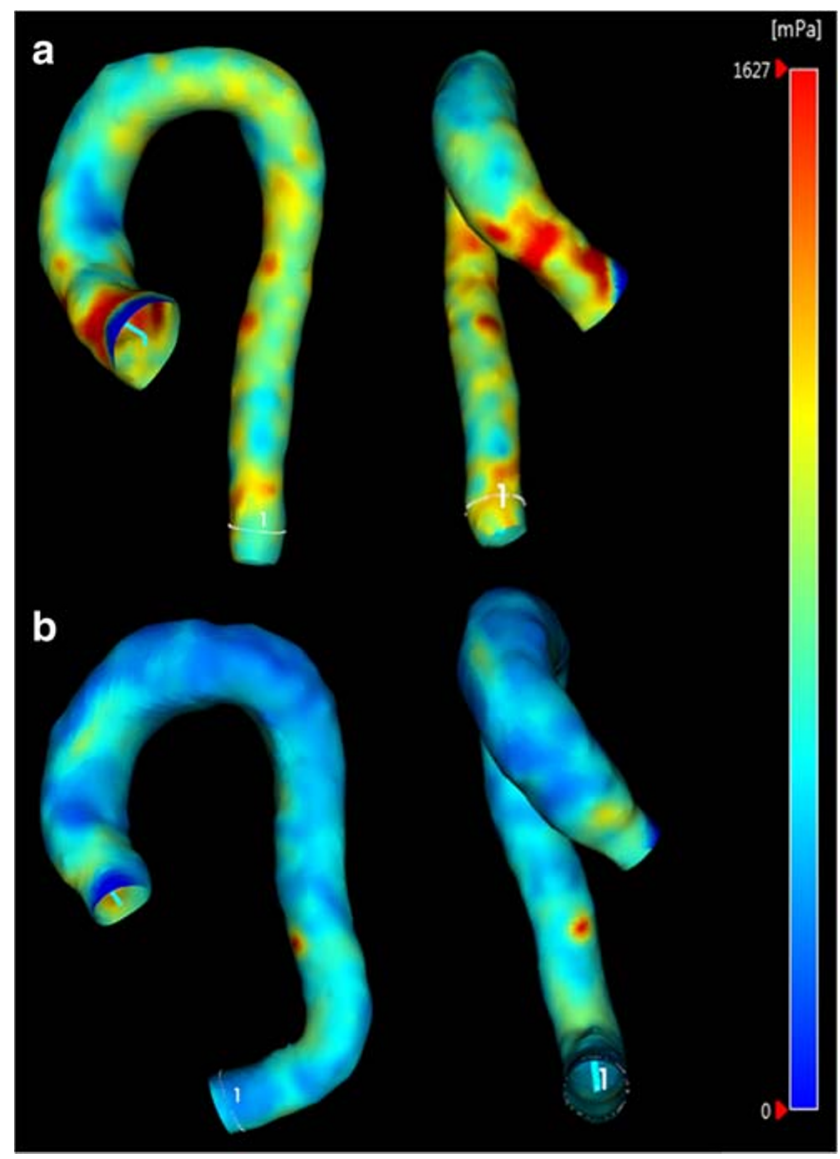

Fig. 5 Illustrative wall shear stress (WSS) images of the dilated AA patients. a Heart-aorta-angle (HAA) of $118^{\circ}$. WSS is clearly increased in the outer curvature of AA (the red areas). b HAA of $132^{\circ}$. WSS is less extensively increased in the outer curvature of AA 


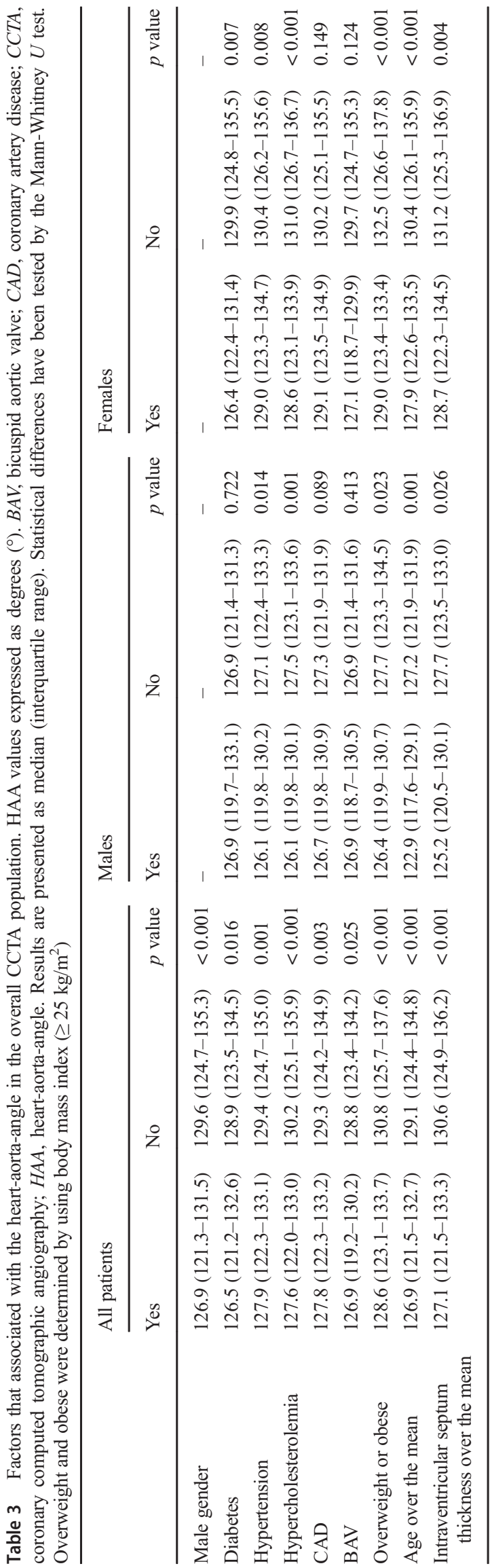

retrospective nature of the study. In addition, a relatively high number of patients had one or more risk factors for cardiovascular diseases and thus, the subpopulation of patients with no risk factors remained relatively small. No follow-up imaging was performed to show causal effects of the smaller HAA on the development of AA dilatation. The presence of obstruction of the left ventricular outflow tract was not registered in CCTA patient population, which might have had an effect on the association between HAA and AA dilatation. However, the presence of the left ventricular outflow tract obstruction is estimated to be very low in this relatively healthy population. Furthermore, the patients in 4D MRI flow study were older and mostly male which limits the comparison with the CCTA patients.

The HAA measurement method, used in this study, has shown to have high intraobserver and interobserver reproducibility in earlier studies [9]. Our study supports the previous findings of high reproducibility of HAA measurements. Since the measurement of HAA is straightforward, fast, and reproducible, it provides an additional tool to analyze CT images of the aorta and heart. The measurement may have a potential as an additional indicator predicting further AA dilatation and stratifying patients for follow-up CT examinations, together with other risk factors such as BAV or genetic risk factors for AA dilatation.

To conclude, the smaller HAA associates significantly with AA dilatation. The smaller HAA increases WSS in the outer curvature of the proximal AA. The clinical relevance of HAA needs to be further verified especially the contributions from the upper abdominal visceral fat and aortic elongation. Thus, further clinical and imaging follow-up studies are needed to verify the possible clinical value of this index.

Funding information Open access funding provided by University of Eastern Finland (UEF) including Kuopio University Hospital. This study has received funding by Oiva Vaittinen Will Grant, Aarne Koskelo Foundation, Finnish Society of Angiology, Radiological Society of Finland, Northern Savonia Foundation of the Finnish Cultural Foundation, Finnish Foundation of Cardiovascular Research, Ida Montin Foundation, Mauri and Sirkka Wiljasalo Foundation, and Instrumentarium Science Foundation.

\section{Compliance with ethical standards}

Guarantor The scientific guarantor of this publication is Marja Hedman.

Conflict of interest The authors of this manuscript declare no relationships with any companies whose products or services may be related to the subject matter of the article.

Statistics and biometry Statistician Tuomas Selander kindly provided statistical advice for this manuscript.

Informed consent Written informed consent was not required for CCTA patient population because this was the retrospective study. 
Written informed consent was obtained from 4D flow MRI patients in this study.

Ethical approval Institutional Review Board approval was obtained.

Study subjects or cohorts overlap Some study subjects or cohorts have been previously reported in:

Korhonen M, Mustonen P, Hedman M, Vienonen J, Onatsu J, Vanninen R, et al. Left atrial appendage morphology and relative contrast agent concentration in patients undergoing coronary artery CTA. Clin Radiol 2018 Jul 18.

Kauhanen SP, Saari P, Jaakkola P, Korhonen M, Parkkonen J, Vienonen $\mathrm{J}$, et al. High prevalence of ascending aortic dilatation in a consecutive coronary CT angiography patient population. Eur Radiol 2020 Feb;30 [2]:1079-1087.

Kauhanen SP, Hedman M, Kariniemi E, Jaakkola P, Vanninen R, Saari $\mathrm{P}$, et al. Aortic dilatation associates with flow displacement and increased circumferential wall shear stress in patients without aortic stenosis: a prospective clinical study. J Magn Reson Imaging 2019 Jul;50 [1]:136-145.

\section{Methodology}

- Retrospective

- Diagnostic or prognostic study

- Performed at one institution

Open Access This article is licensed under a Creative Commons Attribution 4.0 International License, which permits use, sharing, adaptation, distribution and reproduction in any medium or format, as long as you give appropriate credit to the original author(s) and the source, provide a link to the Creative Commons licence, and indicate if changes were made. The images or other third party material in this article are included in the article's Creative Commons licence, unless indicated otherwise in a credit line to the material. If material is not included in the article's Creative Commons licence and your intended use is not permitted by statutory regulation or exceeds the permitted use, you will need to obtain permission directly from the copyright holder. To view a copy of this licence, visit http://creativecommons.org/licenses/by/4.0/.

\section{References}

1. Erbel R, Aboyans V, Boileau C et al (2014) 2014 ESC guidelines on the diagnosis and treatment of aortic diseases: document covering acute and chronic aortic diseases of the thoracic and abdominal aorta of the adult. The Task Force for the Diagnosis And Treatment Of Aortic Diseases of the European Society of Cardiology (ESC). Eur Heart J 35(41):2873-2926

2. Roman MJ, Devereux RB, Kramer-Fox R, O'Loughlin J (1989) Two-dimensional echocardiographic aortic root dimensions in normal children and adults. Am J Cardiol 64(8):507-512

3. Johnston KW, Rutherford RB, Tilson MD, Shah DM, Hollier L, Stanley JC (1991) Suggested standards for reporting on arterial aneurysms. Subcommittee on reporting standards for arterial aneurysms, ad hoc committee on reporting standards, Society for Vascular Surgery and North American Chapter, International Society for Cardiovascular Surgery. J Vasc Surg 13(3):452-458
4. Rogers IS, Massaro JM, Truong QA et al (2013) Distribution, determinants, and normal reference values of thoracic and abdominal aortic diameters by computed tomography (from the Framingham Heart Study). Am J Cardiol 111(10):1510-1516

5. Adriaans BP, Heuts S, Gerretsen S et al (2018) Aortic elongation part I: the normal aortic ageing process. Heart 104(21):1772-1777

6. Malmivuo J, Plonsey R (1995) Bioelectromagnetism. Oxford University Press. https://doi.org/10.1093/acprof:oso/ 9780195058239.001.0001

7. Comstock CH (1987) Normal fetal heart axis and position. Obstet Gynecol 70(2):255-259

8. Sathananthan G, Zahid S, Aggarwal G, Chik W, Friedman D, Thiagalingam A (2015) Cardiac orientation: is there a correlation between the anatomical and the electrical axis of the heart? Br J Cardiol. https://doi.org/10.5837/bjc.2015.016

9. Kwon DH, Smedira NG, Popovic ZB et al (2009) Steep left ventricle to aortic root angle and hypertrophic obstructive cardiomyopathy: study of a novel association using three-dimensional multimodality imaging. Heart 95(21):1784-1791

10. Kauhanen SP, Hedman M, Kariniemi E et al (2019) Aortic dilatation associates with flow displacement and increased circumferential wall shear stress in patients without aortic stenosis: a prospective clinical study. J Magn Reson Imaging 50(1):136-145

11. van Ooij P, Markl M, Collins JD et al (2017) Aortic valve stenosis alters expression of regional aortic wall shear stress: new insights from a 4-dimensional flow magnetic resonance imaging study of 571 subjects. J Am Heart Assoc. https://doi.org/10.1161/JAHA. 117.005959

12. Korhonen M, Mustonen P, Hedman M et al (2018) Left atrial appendage morphology and relative contrast agent concentration in patients undergoing coronary artery CTA. Clin Radiol 73(11): 982.e17-982.e26

13. Kauhanen SP, Saari P, Jaakkola et al (2020) High prevalence of ascending aortic dilatation in a consecutive coronary CT angiography patient population. Eur Radiol 30(2):1079-1087

14. Dyverfeldt P, Bissell M, Barker AJ et al (2015) 4D flow cardiovascular magnetic resonance consensus statement. J Cardiovasc Magn Reson. https://doi.org/10.1186/s12968-015-0174-5

15. Hannuksela M, Lundqvist S, Carlberg B (2006) Thoracic aortadilated or not? Scand Cardiovasc J 40(3):175-178

16. Nuttall FQ (2015) Body mass index: obesity, BMI, and health: a critical review. Nutr Today 50(3):117-128

17. Mulè G, Nardi E, Morreale M et al (2017) The relationship between aortic root size and hypertension: an unsolved conundrum. Adv Exp Med Biol 956:427-445

18. Griepp RB, Ergin MA, Galla JD et al (1999) Natural history of descending thoracic and thoracoabdominal aneurysms. Ann Thorac Surg 67(6):1927-1958

19. Raffort J, Lareyre F, Clément M, Hassen-Khodja R, Chinetti G, Mallat Z (2018) Diabetes and aortic aneurysm: current state of the art. Cardiovasc Res 114(13):1702-1713

20. Després J (2006) Abdominal obesity: the most prevalent cause of the metabolic syndrome and related cardiometabolic risk. Eur Heart J Supp 8(suppl_B):B4-B12

Publisher's note Springer Nature remains neutral with regard to jurisdictional claims in published maps and institutional affiliations. 